\title{
Instruments used to assess oral health-related quality of life of children
}

\author{
Hanan Raja Aljohani ${ }^{1 *}$, Khalid Mubarak Alshammari², Ahmed Mohammed Shaikh ${ }^{3}$, \\ Turki Nasser Alotaibi², Abdullah Ali Alshehri ${ }^{4}$, Abdullah Ali Alsaikhan ${ }^{5}$, \\ Alanoud Meteb Almalky6, Majed Mohammad Zahran ${ }^{7}$, Anaan Ibrahim Bushnag ${ }^{8}$, \\ Fatimah Ismail Alyami ${ }^{9}$, Sarah Khalid Alanzi ${ }^{10}$
}

\author{
${ }^{1}$ Department of Pediatric Dentistry, King Abdullah Medical Complex, Jeddah, Saudi Arabia \\ ${ }^{2}$ Ministry of Health, Hail, Saudi Arabia \\ ${ }^{3}$ College of Dentistry, Imam Abdulrahman Bin Faisal University, Dammam, Saudi Arabia \\ ${ }^{4}$ Al-Moasah Clinics, Abha, Saudi Arabia \\ ${ }^{5}$ Ministry of Interior, Riyadh, Saudi Arabia \\ ${ }^{6}$ Primary Healthcare, Ministry of Health, Tabuk, Saudi Arabia \\ ${ }^{7}$ BioLife Dental Clinics, Jeddah, Saudi Arabia \\ ${ }^{8}$ Ministry of Health, Turaif, Saudi Arabia \\ ${ }^{9}$ Primary Healthcare, Ministry of Health, AlBaha, Saudi Arabia \\ ${ }^{10}$ Dental Department, Dammam Medical Complex, Dammam, Saudi Arabia
}

Received: 22 December 2021

Accepted: 06 January 2022

\author{
*Correspondence: \\ Dr. Hanan Raja Aljohani, \\ E-mail: Hananaljohani99@gmail.com
}

Copyright: (C) the author(s), publisher and licensee Medip Academy. This is an open-access article distributed under the terms of the Creative Commons Attribution Non-Commercial License, which permits unrestricted non-commercial use, distribution, and reproduction in any medium, provided the original work is properly cited.

\begin{abstract}
To evaluate oral health-related quality of life $(\mathrm{OHRQOL})$ in children, both the clinical indices and parenteral reporting can be used to achieve this effect. The present review provides evidence regarding the currently available instruments to assess the OHRQoL in children. Many instruments were reported, and some were designated for preschoolers. In contrast, others for schoolchildren and adolescents, and some researchers reported that some tools could be used to assess OHRQoL in different age groups. Moreover, some tools can also be used to assess the OHRQoL of children based on their parent's perspectives. The POQL, FIS, PedsQL ${ }^{\mathrm{TM}}$ oral health scale ${ }^{\mathrm{TM}}$, and P-CPQ are the only modalities that can effectively assess OHRQoL in all adolescents and all childhood cycles. Moreover, the OHRQoL hypodontia questionnaire, MIQ, IFAQ, DFTO, CPQ11-14, CPQ8-10, child-OIDP, child-OHIP, and child-DPQ are the currently available tools that can be used to assess OHRQoL in adolescents and schoolchildren. Regarding the assessment of OHRQoL in preschoolers, it has been shown that the only available tools for this context include the SOHO-5, OH-ECQOL, Michigan-OHRQoL, ECOHIS, and DDQ questionnaires.
\end{abstract}

Keywords: Quality of life, Oral health, Survey, Questionnaire, OHRQoL, Children, Dental health

\section{INTRODUCTION}

Although many advances have been recently introduced to the field of dentistry, estimates still indicate that oral diseases still represent a major health problem, being highly prevalent in different global communities. ${ }^{1-3}$ Many complications can occur secondary to oral health conditions, including systemic events. Children can also be affected, and their quality of life might be remarkably impaired. ${ }^{4-6}$ To evaluate oral health-related quality of life (OHRQoL) in children, both the clinical indices and parenteral reporting can be used to achieve this effect. ${ }^{6}$

OHRQoL can be defined as a multifactorial parameter that can be used to assess the patient's self of sense, satisfaction, and expectations of care, functional wellbeing, and oral health. ${ }^{6}$ Therefore, many researchers 
focused their efforts on optimizing the method of reporting of the different domains of OHRQoL in children, whether based on self-reporting from patients or their caregivers. In this context, many modalities were reported in the literature with variable characteristics and advantages, and some of them are designated for specific age groups. In contrast, others were developed for different children age groups. ${ }^{7,8}$ In the present literature review, we will formulate evidence regarding the different instruments that have been reported in the literature to assess OHRQoL in children.

\section{LITERATURE REVIEW}

This literature review is based on an extensive literature search in Medline, Cochrane, and EMBASE databases on which was performed $3^{\text {rd }}$ December 2021 using the medical subject headings (MeSH) or a combination of all possible related terms, according to the database. To avoid missing poetential studies, a further manual search for papers was done through Google Scholar, while the reference lists of the initially included papers. Studies discussing instruments used to assess oral health-related quality of life of children were screened for useful information, with no limitations posed on date, language, age of participants, or publication type.

\section{DISCUSSION}

The research aim is the main determinant and different parameters to assess OHRQoL in children. Accordingly, the scale items should be as specific as possible and should only contain items aiming to assess the OHRQoL among children. In this context, items that cannot discriminate between children and cannot assess the ORHRQoL based on a related condition should be excluded from the scale. Moreover, when developing a novel tool, it should be noted that sensitive-to-change items are much required to assess the impact of an intervention or a treatment modality, especially when conducting clinical trials.

Among the different studies in the literature, evidence shows that many OHRQoL measures have been validated in this context. However, it should be noted that many concerns are usually associated when developing these modalities. This has been attributed to the fact that the assessment of OHRQoL is remarkably age-specific. Therefore, assessing these modalities in children is significantly different from in adults. Nevertheless, studies indicate that many tools have been validated for assessing OHRQoL in children. These include the child oral health impact profile (COHIP), Child oral impacts on daily performances (Child-OIDP), pediatric oral healthrelated quality of life (POQL), the early childhood oral health impact score (ECOHIS), the child perception questionnaire (CPQ), the parental-caregiver perceptions questionnaire (P-CPQ), family impact scale (FIS), the pediatric quality of life inventory ${ }^{\mathrm{TM}}$ oral health scale ${ }^{\mathrm{TM}}$ (PedsQL ${ }^{\mathrm{TM}}$ oral health scale $^{\mathrm{TM}}$ ), oral health-related quality of life for patients with hypodontia (OHRQoL hypodontia), malocclusion impact questionnaire (MIQ), dental freetime tradeoff scale (DFTO), impact of fixed appliances questionnaire (IFAQ), child dental pain questionnaire (Child-DPQ), scale of oral health outcomes for 5-year old children (SOHO-5), early childhood oral health impact scale (ECOHIS), dental discomfort questionnaire (DDQ), oral health-related early childhood quality of life (OH-ECQOL), and Michigan oral healthrelated quality of life (Michigan-OHRQoL). ${ }^{9-26}$

In 2002, the CPQ11-14 was first reported by Jokovic et al and included 37 items to assess OHRQoL in children aged 11-14 years old. ${ }^{23}$ The CPQ8-10 was first reported in 2004 and targeted children aged 8-10 years old and consisted of 25 items. The Child-OIDP was also first reported in 2004 and consisted of 8 items, targeting children 10-12 years of age. ${ }^{14,27}$ Moreover, the ECOHIS tool was developed in 2007 and aimed at assessing the OHRQoL in children at 3-5 years old. ${ }^{20}$ The COHIP questionnaire was first reported in 2007 and consisted of 34 items and targeted children aged 7-18 years old. ${ }^{15}$ Finally, the POQL was developed and first reported in 2011 and consisted of 20 items that targeted children aged 2-12 years of age. ${ }^{9}$ It should be noted that the POQL, FIS, PedsQL ${ }^{\mathrm{TM}}$ oral health scale ${ }^{\mathrm{TM}}$, and P-CPQ are the only modalities that can be effectively used for the assessment of OHRQoL in all adolescents and all childhood cycles. Moreover, the OHRQoL hypodontia questionnaire, MIQ, IFAQ, DFTO, CPQ11-14, CPQ8-10, child-OIDP, childOHIP, and child-DPQ are the currently available tools that can be used to assess OHRQoL in adolescents and schoolchildren. Regarding the assessment of OHRQoL in preschoolers, it has been shown that the only available tools for this context include the SOHO-5, OH-ECQOL, Michigan-OHRQoL, ECOHIS, and DDQ questionnaires.

Among the different reported scales, it can be observed that the first measurement to assess OHRQoL in children has been the CPQ. ${ }^{28}$ Besides, as we mentioned, there are two forms of ages for this questionnaire. However, it is not clear whether these two forms are continuous and complement each other or not. It has been furtherly shown that the ECOHIS scale can be used to assess OHRQoL in preschool children based on the evaluation of different parameters and conditions, including craniofacial, orthodontic, and dental conditions based on reports of caregivers of these children. ${ }^{20}$ The POQL score has been reported to be a more novel questionnaire that can be better used for both school-aged and preschool children. ${ }^{9}$ It has been shown that the questionnaire can significantly identify caries and other dental conditions among both vulnerable and general conditions. When this tool was first reported, it was meant to evaluate OHRQoL among 12-year-old children in Thailand. ${ }^{14}$ However, evidence from multiple investigations shows that the tool has been extensively used among different global communities and has been translated into many languages. Accordingly, it has been reported among population-based investigations and mainly aims at 
assessing the impact of oral disorders on everyday activities and different parameters of OHRQoL. ${ }^{27}$

It should be noted that both the positive and negative health impact of the different oral conditions was first reported in combination using the COHIP tool. ${ }^{29}$ Accordingly, relevant studies indicated that it could be effectively used to exclude the presence or impact of an oral condition and assess the enhanced well-being and positive attributes secondary to applying adequate care. ${ }^{30-}$ 32 Different versions were reported among the different studies in the literature for this tool, including a short form and teacher, caregiver, and child versions. Evidence indicates that assessment and utilization of information regarding OHRQoL can be effectively conducted via short forms in a quick and facilitated pattern. It has been furtherly shown that all of the reported instruments have been used in different languages and were used in many clinical studies. ${ }^{29}$

A previous systematic review compared the different used for assessment of OHRQoL in different age groups, including adolescents, schoolchildren, and preschoolers, in addition to scales that assessed OHRQoL among mixed populations of children. ${ }^{7}$ Based on the standard criteria of the evaluating measures of patient-reported outcomes (EMPRO) tool, it has been shown that the CPQ11-14 for schoolchildren and the ECOHIS in preschoolers are the most highly rated scales based on evidence from different studies. Moreover, it has been shown that the impact of oral health and related conditions on the family of the affected children was also reported to be best assessed using the FIS scale. However, it should also be noted that other tools can also be recommended based on their performance among the various relevant studies. These might include the P-CPQ and the POQL for any age and the child-OHIP and child-OIDP in schoolchildren. On the other hand, it has been reported that instruments designated to assess hypodontia and malocclusion were below the threshold of criteria while CPQ8-10 in schoolchildren and the SOHO-5 in preschoolers scored just above the threshold, which makes them also valid to be used for evaluation of OHRQoL in children. ${ }^{7}$

Although most of the reported tools in the literature are adequately validated for measuring OHRQoL among preschoolers, it has been shown that only the ECOHIS is most frequently correlated with acceptable significant levels of interpretability, responsiveness, and reliability. In another context, although it has been reported that the SOHO-5 has a high responsiveness rate, evidence indicates that more data is still needed for further evaluation of the interpretability and reliability of the modality. ${ }^{33,34}$ Other advantages were also reported for the ECOHIS system, including having a section for assessing the impact of children's dental conditions on the quality of life of their families and being translated to at least 14 global languages among the different international investigations. Evidence indicates that both the SOHO-5 and ECOHIS tools are widely used as generic modalities for the assessment of OHRQoL among children based on their abilities to evaluate dental health diseases and also being able to evaluate several oral pathogens. ${ }^{4,35}$

Regarding the tools that have been validated for assessment of OHRQoL among adolescents, evidence shows that the most significant tools include the childOHIP, the child-OIDP, and the CPQ11-14, having the highest scores on the EMPRO assessment scale, in addition to being the most favorable results of responsiveness, validity, reliability, and conceptual model. It has been furtherly shown that a high EMPRO score for interpretability was also reported for the CPQ11-14, which has also been reported to be valid for evaluating many conditions, including craniofacial disorders, malocclusion, dental fluorosis, enamel defects, and caries. ${ }^{23,36-41}$ This tool is long (consists of 34 items). However, it has been shown that using the short version of the tool can allow for a facilitated evaluation and easy interpretation.

Among the different studies in the literature, evidence also indicates that the POQL, P-CPQ, and the FIS are the most validated measures for evaluation of OHRQoL among children of any age group. However, it should be considered that the POQL is only used for evaluating dental caries, the P-CPQ is used for evaluating OHRQoL of children based on their parent's perspectives, and the FIS also evaluates the impact on the quality of life of families. Moreover, research indicates that the P-CPQ and FIS tools were originally developed to assess OHRQoL among children aged 6-14 years old. However, it has been shown that these tools can be furtherly used to assess these outcomes in children $\geq 3$ years old based on their psychometric characteristics. Besides, they are present in five different languages, and short segments of these tools were also validated among different studies in the literature for evaluating the OHRQoL in different conditions. These include orthodontic treatment, dental fluorosis, oro-facial conditions, and dental caries. ${ }^{24,26,42,43}$ A previous systematic review also indicates the validity and significance of using $\mathrm{P}-\mathrm{CPQ}$, child-OHIP, and childODIP to assess OHRQoL in children. ${ }^{8}$ It should be noted that the number of studies analyzed in this review was less than the number of investigations analyzed in the review by Zaror et al which showed more favorable results for these instruments regarding interpretability, responsiveness, and reliability. ${ }^{7}$ It should be noted that age is a vital factor that should be considered when screening for OHRQoL in children. Therefore, it is logical that the way of reporting OHRQoL among children is different based on age and how they interpret this data.

Among the different studies in the literature, it has been reported that the only instrument that can adequately assess OHRQoL with age variations without missing any essential age-related information is the PedsQL-OH tool. ${ }^{25,44}$ However, it should be noted that self-reporting in children can be adequately obtained when the child is 
around six years old. This is because children usually compare their personality traits and physical features and begin abstract thinking by this age. In this context, a previous SOHO-5-based investigation reported that the reported aspects of OHRQoL among children and their caregivers were remarkably significant. ${ }^{45}$ However, it should be noted that not all tools can assess such differences as we previously mentioned. Accordingly, further research is needed to adapt such assessment strategies adequately.

\section{CONCLUSION}

The present review provides evidence regarding the currently available instruments to assess the OHRQoL in children. Many instruments were reported, and some were designated for preschoolers. In contrast, others for schoolchildren and adolescents, and some researchers reported that some tools could be used to assess OHRQoL in different age groups. Moreover, some tools can also be used to assess the OHRQoL of children based on their parent's perspectives. These include FIS, P-CPQ, and the CPQ questionnaires. Evidence indicates the validity of these instruments, which can also be easily applied and adapted among different languages and communities.

Funding: No funding sources

Conflict of interest: None declared

Ethical approval: Not required

\section{REFERENCES}

1. Kassebaum NJ, Smith AGC, Bernabé E. Global, Regional, and National Prevalence, Incidence, and Disability-Adjusted Life Years for Oral Conditions for 195 Countries, 1990-2015: A Systematic Analysis for the Global Burden of Diseases, Injuries, and Risk Factors. J Dental Res. 2017;96(4):380-7.

2. Aldrigui JM, Jabbar NS, Bonecker M, Braga MM, Wanderley MT. Trends and associated factors in prevalence of dental trauma in Latin America and Caribbean: a systematic review and meta-analysis. Community Dentistry Oral Epidemiol. 2014;42(1):30-42.

3. Hoffmeister L, Moya P, Vidal C, Benadof D. Factors associated with early childhood caries in Chile. Gac Sanit. 2016;30(1):59-62.

4. Abanto J, Carvalho TS, Mendes FM, Wanderley MT, Bönecker M, Raggio DP. Impact of oral diseases and disorders on oral health-related quality of life of preschool children. Community Dentistry Oral Epidemiol. 2011;39(2):105-14.

5. Kragt L, Dhamo B, Wolvius EB, Ongkosuwito EM. The impact of malocclusions on oral health-related quality of life in children-a systematic review and meta-analysis. Clin Oral Investigations. 2016;20(8):1881-94.

6. Sischo L, Broder HL. Oral health-related quality of life: what, why, how, and future implications. J Dental Res. 2011;90(11):1264-70.
7. Zaror C, Pardo Y, Espinoza-Espinoza G. Assessing oral health-related quality of life in children and adolescents: a systematic review and standardized comparison of available instruments. Clin Oral Investigations. 2019;23(1):65-79.

8. Genderson MW, Sischo L, Markowitz K, Fine D, Broder HL. An overview of children's oral healthrelated quality of life assessment: from scale development to measuring outcomes. Caries Res. 2013;47(1):13-21.

9. Huntington NL, Spetter D, Jones JA, Rich SE, Garcia RI, Spiro A, 3rd. Development and validation of a measure of pediatric oral health-related quality of life: the POQL. J Public Health Dentistry. 2011;71(3):185-93.

10. Akram AJ, Jerreat AS, Woodford J, Sandy JR, Ireland AJ. Development of a condition-specific measure to assess quality of life in patients with hypodontia. Orthodontics Craniofacial Res. 2011;14(3):160-7.

11. Patel N, Hodges SJ, Hall M, Benson PE, Marshman Z, Cunningham SJ. Development of the Malocclusion Impact Questionnaire (MIQ) to measure the oral health-related quality of life of young people with malocclusion: part 1 - qualitative inquiry. J Orthodontics. 2016;43(1):7-13.

12. Mandall NA, Vine S, Hulland R, Worthington HV. The impact of fixed orthodontic appliances on daily life. Community Dental Health. 2006;23(2):69-74.

13. Jokovic A, Locker D, Tompson B, Guyatt G. Questionnaire for measuring oral health-related quality of life in eight- to ten-year-old children. Pediatr Dentistry. 2004;26(6):512-8.

14. Gherunpong S, Tsakos G, Sheiham A. Developing and evaluating an oral health-related quality of life index for children; the CHILD-OIDP. Community Dental Health. 2004;21(2):161-9.

15. Broder HL, McGrath C, Cisneros GJ. Questionnaire development: face validity and item impact testing of the Child Oral Health Impact Profile. Community Dentistry Oral Epidemiol. 2007;35(1):8-19.

16. Barrêtto ER, Paiva SM, Pordeus IA, Ferreira e Ferreira E. Validation of a child dental pain questionnaire instrument for the self-reporting of toothache in Children. Pediatr Dentistry. 2011;33(3):228-32.

17. Tsakos G, Blair YI, Yusuf H, Wright W, Watt RG, Macpherson LM. Developing a new self-reported scale of oral health outcomes for 5-year-old children (SOHO-5). Health Quality Outcomes. 2012;10:62.

18. Mathur VP, Dhillon JK, Logani A, Agarwal R. Development and validation of oral health-related early childhood quality of life tool for North Indian preschool children. Indian J Dental Res. 2014;25(5):559-66.

19. Filstrup SL, Briskie D, da Fonseca M, Lawrence L, Wandera A, Inglehart MR. Early childhood caries and quality of life: child and parent perspectives. Pediatric Dentistry. 2003;25(5):431-40. 
20. Pahel BT, Rozier RG, Slade GD. Parental perceptions of children's oral health: the Early Childhood Oral Health Impact Scale (ECOHIS). Health Quality Life Outcomes. 2007;5:6.

21. Versloot J, Veerkamp JS, Hoogstraten J. Dental Discomfort Questionnaire: predicting toothache in preverbal children. Eur J Paediatr Dentistry. 2004;5(3):170-3.

22. Fyffe HE, Deery C, Nugent Z, Nuttall NM, Pitts NB. The reliability of two methods of utility assessment in dentistry. Community Dental Health. 1999;16(2):72-9.

23. Jokovic A, Locker D, Stephens M, Kenny D, Tompson B, Guyatt G. Validity and reliability of a questionnaire for measuring child oral-health-related quality of life. J Dental Res. 2002;81(7):459-63.

24. Jokovic A, Locker D, Stephens M, Kenny D, Tompson B, Guyatt G. Measuring parental perceptions of child oral health-related quality of life. J Public Health Dentistry. 2003;63(2):67-72.

25. Steele M, Steele R, Varni J. Reliability and Validity of the PedsQL ${ }^{\text {TM }}$ Oral Health Scale: Measuring the Relationship Between Child Oral Health and HealthRelated Quality of Life. Children's Health Care. 2009;38:228-44.

26. Locker D, Jokovic A, Stephens M, Kenny D, Tompson B, Guyatt G. Family impact of child oral and oro-facial conditions. Community Dentistry Oral Epidemiol. 2002;30(6):438-48.

27. Yusuf H, Gherunpong S, Sheiham A, Tsakos G. Validation of an English version of the Child-OIDP index, an oral health-related quality of life measure for children. Health Quality Life Outcomes. 2006;4:38.

28. Locker D, Jokovic A, Tompson B. Health-related quality of life of children aged 11 to 14 years with orofacial conditions. Cleft Palate-Craniofacial J. 2005;42(3):260-6.

29. Broder HL, Wilson-Genderson M, Sischo L. Reliability and validity testing for the Child Oral Health Impact Profile-Reduced (COHIP-SF 19). J Public Health Dentistry. 2012;72(4):302-12.

30. Son PT, Reda A, Viet DC. Exchange transfusion in the management of critical pertussis in young infants: a case series. Vox Sang. 2021;116(9):976-82.

31. El-Qushayri AE, Ghozy S, Reda A, Kamel AMA, Abbas AS, Dmytriw AA. The impact of Parkinson's disease on manifestations and outcomes of Covid-19 patients: A systematic review and meta-analysis. Rev Med Virol. 2021:e2278.

32. El-Qushayri AE, Dahy A, Reda A, et al. A closer look at the high burden of psychiatric disorders among healthcare workers in Egypt during the COVID-19 pandemic. Epidemiol Health. 2021;43:e2021045.

33. Dibas M, Doheim MF, Ghozy S, Ros MH, El-Helw GO, Reda A. Incidence and survival rates and trends of skull Base chondrosarcoma: A Population-Based study. Clin Neurol Neurosurg. 2020;198:106153.
34. Nguyen TM, Huan VT, Reda A. Clinical features and outcomes of neonatal dengue at the Children's Hospital 1, Ho Chi Minh, Vietnam. J Clin Virol. 2021;138:104758.

35. Gomes MC, Pinto-Sarmento TC, Costa EM, Martins CC, Granville-Garcia AF, Paiva SM. Impact of oral health conditions on the quality of life of preschool children and their families: a cross-sectional study. Health Quality Life Outcomes. 2014;12:55.

36. Wogelius P, Gjørup H, Haubek D, Lopez R, Poulsen S. Development of Danish version of child oralhealth-related quality of life questionnaires (CPQ810 and CPQ11-14). BMC Oral Health. 2009;9:11.

37. O'Brien K, Wright JL, Conboy F, Macfarlane T, Mandall N. The child perception questionnaire is valid for malocclusions in the United Kingdom. Am J Orthodontics Dentofacial Orthopedics. 2006;129(4):536-40.

38. O'Brien C, Benson PE, Marshman Z. Evaluation of a quality of life measure for children with malocclusion. J Orthodontics. 2007;34(3):185-93..

39. Kumar S, Kroon J, Lalloo R, Johnson NW. Psychometric Properties of Translation of the Child Perception Questionnaire (CPQ11-14) in Telugu Speaking Indian Children. PloS One. 2016;11(3):e0149181.

40. Marshman Z, Rodd H, Stern M. An evaluation of the Child Perceptions Questionnaire in the UK. Community dental health. 2005;22(3):151-5.

41. Shin HS, Han DH, Shin MS, Lee HJ, Kim MS, Kim HD. Korean version of child perceptions questionnaire and dental caries among Korean children. PloS one. 2015;10(2):e0116011.

42. Marshman Z, Rodd H, Stem M, Mitchell C, Robinson PG. Evaluation of the Parental Perceptions Questionnaire, a component of the COHQoL, for use in the UK. Community Dental Health. 2007;24(4):198-204.

43. Kumar S, Kroon J, Lalloo R, Johnson NW. Validity and reliability of short forms of parental-caregiver perception and family impact scale in a Telugu speaking population of India. Health Quality Life Outcomes. 2016;14(1):34.

44. Wallander JL, Schmitt M, Koot HM. Quality of life measurement in children and adolescents: issues, instruments, and applications. J Clin Psychol. 2001;57(4):571-85.

45. Abanto J, Tsakos G, Paiva SM, Raggio DP, Celiberti P, Bönecker M. Agreement between children aged 56 years and their mothers in rating child oral healthrelated quality of life. Int $\mathbf{J}$ Paediatr Dentistry. 2014;24(5):373-9.

Cite this article as: Aljohani HR, Alshammari KM, Shaikh AM, Alotaibi TN, Alshehri AA, Alsaikhan AA et al. Instruments used to assess oral healthrelated quality of life of children. Int J Community Med Public Health 2022;9:1051-5. 